\title{
Comparison of the GlideScope and the McGrath method using vascular forceps and a tube exchanger in cases of simulated difficult airway intubation
}

\author{
Jae-Hang Shim, Woo Jae Jeon, Sang Yun Cho, and Gyu Ho Choe \\ Department of Anesthesiology and Pain Medicine, Hanyang University Guri Hospital, Guri, Korea
}

Background: A "difficult airway" can be simulated with an extrication collar, which restricts cervical motion and mouth opening. The purpose of this study is to compare the efficacy of the GlideScope and the McGrath in difficult airway simulation.

Methods: Patients were randomized using computer-generated numbers and were placed into the GlideScope group or the McGrath group. The total intubation time was defined as the time measured from when the anesthesiologist picks up the device to the time at which three successive end-tidal $\mathrm{CO}_{2}$ values are acquired after intubation.

Results: There was no significant difference in total intubation time between the two groups $(73.0 \pm 25.3 \mathrm{sec}$ vs. $72.3 \pm 20.9 \mathrm{sec}, \mathrm{P}=0.92)$. The success rates of the first intubation attempt did not differ between the two groups $(82.8 \%$ vs. $83.3 \%, \mathrm{P}=0.95)$.

Conclusions: Our results suggest that there are no significant differences in the intubations with GlideScope and McGrath using vascular forceps and tube exchangers in difficult intubation scenarios.

Key Words: Airway management, Intratracheal intubation, Laryngoscopes.

\section{Introduction}

The Glidescope ${ }^{\circledR}$ videolaryngoscope (GVL; Verathon Inc., Bothell, WA, USA) is a relatively new device for endotracheal

Corresponding author: Sang Yun Cho, M.D., Ph.D.

Department of Anesthesiology and Pain Medicine, Hanyang University Guri Hospital, 153, Gyeongchun-ro, Guri 11923, Korea

Tel: 82-31-560-2394, Fax: 82-31-563-1731

E-mail: chosy@hanyang.ac.kr

ORCID: http://orcid.org/0000-0002-6593-1960

Received: October 14, 2015.

Revised: 1st, November 9, 2015; 2nd, November 25, 2015.

Accepted: November 27, 2015.

Korean J Anesthesiol 2016 April 69(2): 133-137

http://dx.doi.org/10.4097/kjae.2016.69.2.133 intubation. The Glidescope provides a better view of the larynx than other devices do, and it is therefore useful for managing difficult airways [1]. Similarly, the McGrath ${ }^{\circledR}$ Series 5 (Aircraft Medical, Edinburgh, UK) is a self-contained videolaryngoscope that has not only been used successfully in normal airway management, but also in difficult airway management [2]. A difficult airway can be simulated with an extrication collar, which restricts cervical motion and mouth opening. This difficult airway simulation does not harm the patients [3].

Difficult airways are not commonly encountered clinically. Application of a cervical collar is a well established method for evaluating airway device performance in a more difficult airway scenario. The presence of a cervical collar makes airway management difficult because it restricts the laryngoscopic view. Use of a cervical collar is also less ethically challenging than use of unproven airway devices for simulating difficult airways [4,5].

(c) This is an open-access article distributed under the terms of the Creative Commons Attribution Non-Commercial License (http://creativecommons.org/ licenses/by-nc/4.0/), which permits unrestricted non-commercial use, distribution, and reproduction in any medium, provided the original work is properly cited. 
The purpose of this study is to compare the efficacy of the GlideScope and the McGrath in difficult airway simulation. Our primary objectives were to compare the total tracheal intubation time and the success rates of tracheal intubation by using these two different devices.

\section{Materials and Methods}

The study protocol was approved by the Institutional Review Board, the panel on Human Subjects Research and Ethics Committee. Written informed consent was obtained from patients before enrolment in the study. Participants were adult patients (aged 18-65 years) who were scheduled for elective surgeries and were classified as American Society of Anesthesiologists physical status I, II or III. Exclusion criteria included the following: history of difficult intubation; laryngeal surgery; radiotherapy to the larynx; tracheal, respiratory or cardiovascular disease; patients expected to have esophago-tracheal reflux; patients who had a loose tooth; patients with a Mallampati classification of III or IV; and patients with a body mass index $>35 \mathrm{~kg} / \mathrm{m}^{2}$.

Patients were interviewed before surgery to evaluate their condition and Mallampati scores. Patients were randomized into the GlideScope group or the McGrath group according to computer-generated numbers.

Once the patient arrived in the operation room, electrocar- diography, pulse oximetry, non-invasive blood pressure and end tidal $\mathrm{CO}_{2}$ were monitored. A nurse who was not involved in the study opened a sealed, opaque envelope that contained the patient's number and group information immediately before induction of general anesthesia. The depth of anesthesia was measured with a bispectral index (BIS) monitor (A-2000TM, version 3.3, Aspect Medical System Inc., Newton, MA, USA). Patients were allowed to breathe $100 \%$ oxygen for three minutes to remove all the nitrogen gas from their airways. After denitrogenation was complete, $3-5 \mathrm{mg} / \mathrm{kg}$ sodium pentothal was intravenously injected and 6-8 vol\% of the inhaled anesthetic agent desflurane was administered. When the BIS index was below 60, the Philadelphia Patriot ${ }^{\circledR}$ cervical collar (Philadelphia Cervical Collar Co., Thorofare, NJ, USA) was placed and secured to the patient's head with an elastic bandage. To induce muscle relaxation, esmeron was injected. A nerve stimulator was used to monitor the train-of-four reactions. When the train-of-four reaction was confirmed to be zero, the laryngoscope was directly inserted to view the glottis. Before advancing the laryngoscope, the size of the mouth opening was measured. When the BIS index fell below 60 again with continuous desflurane administration, the GlideScope or McGrath videolaryngoscope was used to intubate patients in the GlideScope and McGrath groups, respectively. With regard to past simulation studies of difficult airway intubation with the GlideScope, we used a tube exchanger

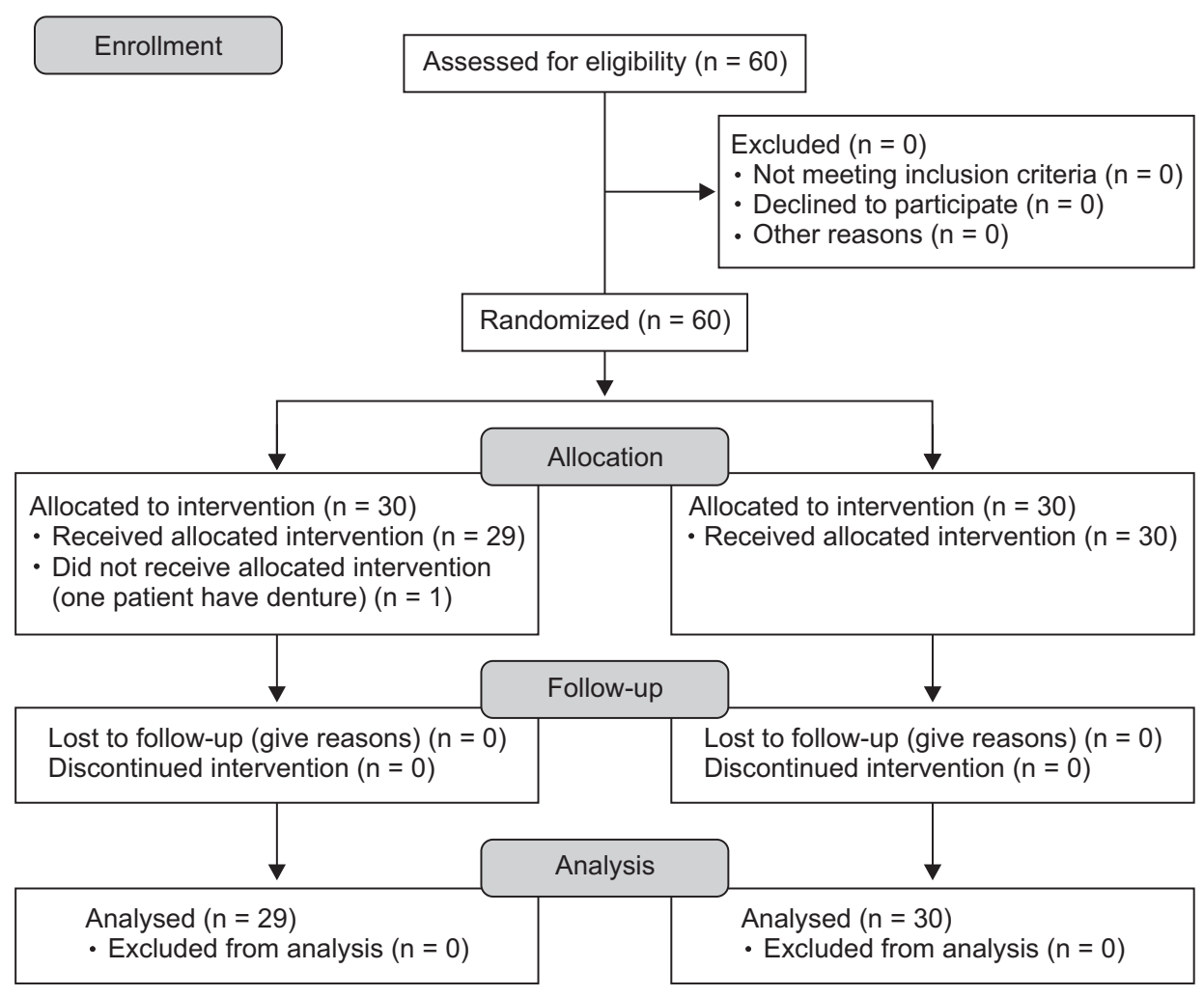

Fig. 1. Study flow diagram 
and vascular forceps instead of a stylet used in the usual method [5].

A glottis view was obtained with insertion of each device. The total intubation time was defined as the amount of time taken by the anesthesiologist to pick up the device until he/she obtained three successive end-tidal $\mathrm{CO}_{2}$ waves after intubation. If the first attempt was unsuccessful, oxygen and desflurane were administered until the BIS index was once again below 60 . If the intubation was unsuccessful after three attempts, it was considered a failed intubation. In case of a failed intubation, the cervical collar was removed and the patient was intubated normally. After extubation, we checked for blood in the intubation tube, the presence of damaged oral tissue, damage to teeth and other complications. We met the patients one hour and 24 hours after surgery to enquire whether they were suffering from a sore throat.

To estimate the sample size needed, we used the average and standard deviation obtained from a previous study [5]. The

Table 1. Characteristics and Airway Data of Patients Undergoing Tracheal Intubation Using the GlideScope or the McGrath Method with a Tube Exchanger and Forceps

\begin{tabular}{lcc}
\hline & $\begin{array}{c}\text { GlideScope } \\
(\mathrm{n}=29)\end{array}$ & $\begin{array}{c}\text { McGrath } \\
(\mathrm{n}=30)\end{array}$ \\
\hline ASA physical status (I/II/III) & $11 / 18 / 0$ & $18 / 11 / 1$ \\
Age $(\mathrm{yr})$ & $47.2 \pm 12.6$ & $44.5 \pm 12.9$ \\
Sex $(\mathrm{M} / \mathrm{F})$ & $14 / 15$ & $14 / 16$ \\
Weight $(\mathrm{kg})$ & $67.6 \pm 9.8$ & $67.2 \pm 12.1$ \\
Height $(\mathrm{cm})$ & $164.9 \pm 8.2$ & $165.7 \pm 9.1$ \\
Body mass index $\left(\mathrm{kg} / \mathrm{m}^{2}\right)$ & $24.8 \pm 2.5$ & $24.4 \pm 3.3$ \\
Thyromental distance $(\mathrm{cm})$ & $6.6 \pm 0.8$ & $6.9 \pm 0.6$ \\
Neck circumference $(\mathrm{cm})$ & $38.1 \pm 4.1$ & $37.9 \pm 4.0$ \\
Mouth opening & $3.9 \pm 0.5$ & $3.8 \pm 0.4$ \\
without a cervical collar $(\mathrm{cm})$ & &
\end{tabular}

Values are expressed as raw numbers or means \pm SD. ASA: American Society of Anesthesiologists. average and standard deviation were 66.1 and 15.5 seconds, respectively. We needed 28.8 patients to detect a $20 \%$ difference between the groups, but assuming a $2 \%$ drop out rate, 60 patients were required in our study. Our tests were two tailed with $\alpha=0.05$ and $\beta=90 \%$. For our statistical analyses, we used the Statistical Package for Social Sciences 17.0 for Windows (SPSS Inc., Chicago, IL, USA). Unpaired t-tests were used to compare the numerical data between the two groups. Kaplan-Meier plots were generated to compare the total intubation times across the two groups. Nonparametric data were analyzed with the $\chi^{2}$ test and Fisher's exact test. Hemodynamic changes were analyzed with Kruskal-Wallis one way analysis of variance with a Dunn's multiple comparison test. Statistical significance was defined as a $\mathrm{P}$ value $<0.05$.

\section{Results}

Sixty patients were enrolled in our study. One patient in the GlideScope group was excluded in the late stage of the experiment because the patient's false teeth were not detected during the pre-operative meeting (Fig. 1). The size of the mouth opening did not differ between the two groups before or after cervical collar application (Tables 1 and 2). However, there was a change in the size of the mouth opening in each respective group from before cervical collar application to after cervical collar application. There was no difference between the two groups in the glottis view obtained by the laryngoscope after applying the cervical collar. The glottis view simulations that were graded higher than III were expected to be difficult intubations (Table 2). Glottis views obtained by the videolaryngoscope after applying the cervical collar showed a grade of I and II. There was only one case of grade III in the McGrath group. Therefore, we expected that intubation would be easier with the videolaryngoscope. There were no differences between the two groups in the number of attempts or success rates ( $82.8 \%$ vs. $83.3 \%)$ (Table 2$)$. The total

Table 2. Characteristics of Intubation with the GlideScope and McGrath with a Tube Exchanger and Forceps and the Associated Complications

\begin{tabular}{lccc}
\hline & $\begin{array}{c}\text { GlideScope } \\
(\mathrm{n}=29)\end{array}$ & $\begin{array}{c}\text { McGrath } \\
(\mathrm{n}=30)\end{array}$ & P value \\
\hline Mouth opening with collar $(\mathrm{cm})$ & $0.9 \pm 0.3$ & $1.1 \pm 0.4$ & 0.30 \\
Laryngoscopic view (grade $1 / 2 / 3 / 4)$ & $0 / 0 / 15 / 14$ & $0 / 0 / 16 / 14$ & 0.90 \\
Videoscopic view (grade 1/2/3/4) & $21 / 8 / 0 / 0$ & $19 / 10 / 1 / 0$ & 0.52 \\
Total intubation time (seconds) & $73.0 \pm 25.3$ & $72.3 \pm 20.9$ & 0.92 \\
Intubation attempts (1/2/3) & $24 / 4 / 2$ & $25 / 3 / 2$ & 0.79 \\
First attempt success rate & $24 / 29(82.8 \%)$ & $25 / 30(83.3 \%)$ & 0.95 \\
Sore throat in the recovery room & $3 / 29(10.3 \%)$ & $4 / 30(13.3 \%)$ & 0.72 \\
Sore throat at 24 h & $5 / 29(17.2 \%)$ & $2 / 30(6.7 \%)$ & 0.21 \\
Dental injury & 0 & 0 & 0.21 \\
Blood on the tube & $5 / 29(17.2 \%)$ & $2 / 30(6.7 \%)$ & \\
\hline
\end{tabular}

Values are expressed as raw numbers, proportions, or means \pm SD. 


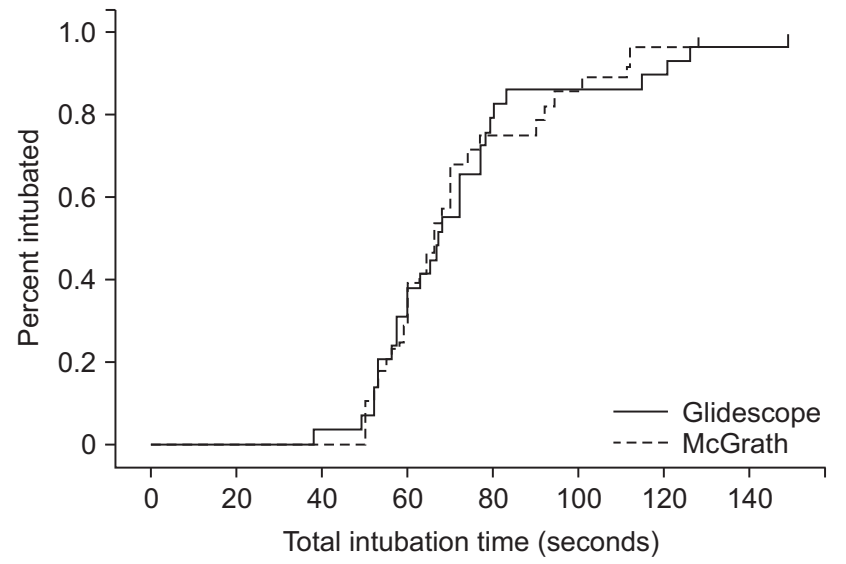

Fig. 2. Kaplan-Meier plot showing the percentage of patients intubated as time progressed. Total intubation times was not significantly different between two groups.

intubation times were also not significantly different between two groups (Fig. 2).

There was no difference in the frequency of sore throat at any time after surgery between the two groups. There were also no differences in the frequency of tooth injury or blood-stained intubation tubes between the two groups.

\section{Discussion}

We found that application of a cervical collar can simulate an airway that is difficult to intubate. There were no significant differences in the total intubation time and success rate between the GlideScope and the McGrath in simulated difficult airway scenarios.

The frequency of difficult airways has been reported to range from $0.8-7.8 \%$ at the time of endotracheal intubation [6-8], and airways requiring more than three attempts at intubation account for $0.9-1.9 \%$ of cases [9]. Since difficult airways are not frequently encountered clinically, it is challenging to establish the techniques that are successful in these situations. Therefore, many simulations of difficult airways have been studied. One way to simulate a difficult airway is to apply a cervical collar [1,3-5]. The use of a cervical collar is a well-established method to immobilize the cervical spine and restrict mouth opening [3-5]. Similar to previous studies, we were able to establish dif- ficult airway simulations with a cervical collar. It was difficult to intubate such experimental airways because of various parameters such as mouth opening $<1.5 \mathrm{~cm}$, glottis views showing grades higher than III and with $47.5 \%$ higher than grade IV. Many studies have compared the GlideScope and McGrath methods. Savoldelli et al. [10] demonstrated that the McGrath method provides better laryngeal exposure than does the GlideScope in a manikin study of a difficult airway. However, there were no differences in performance between these two devices in morbidly obese patients [11]. Jeon et al. [12] found that the intubation time in normal airway patients is shorter with the GlideScope than with the McGrath method. However, the glottis views were not different between the two devices. Our findings cannot be easily compared to previous findings because we simulated difficult airway situations with a cervical collar. There were no differences in total intubation time, success rate of the first intubation attempts or glottis views between the GlideScope and the McGrath. There was only one case of intubation failure in the McGrath group because the blade (size \#4) was too short to provide the laryngeal view and we did not have the next blade size (size \#5) that was needed. Many assist devices used in videolaryngoscopic intubation were studied in the setting of difficult airway simulation. In a previous study, we suggested that it was appropriate to use vascular forceps and a tube exchanger with the GlideScope.

This study has several limitations. Firstly, the attending anesthesiologists were not blinded to the devices. In addition, the attending anesthesiologists were more familiar with the GlideScope than with the McGrath method. Finally, although we simulated difficult airway situations, our simulations cannot truly represent the reality of such a situation. This is especially true because we excluded the patients who had risk factors that can make intubation more difficult. More research is needed to verify our findings.

This is the first study to compare the efficacy of the GlideScope and McGrath videolaryngoscopes in difficult airways induced by application of a cervical collar, and assisted by vascular forceps and a tube exchanger. Our results suggest that there are no significant differences in the intubations with GlideScope and McGrath using vascular forceps and tube exchangers in difficult intubation scenarios.

\section{References}

1. Bathory I, Frascarolo P, Kern C, Schoettker P. Evaluation of the GlideScope for tracheal intubation in patients with cervical spine immobilisation by a semi-rigid collar. Anaesthesia 2009; 64: 1337-41.

2. Shippey B, Ray D, McKeown D. Use of the McGrath videolaryngoscope in the management of difficult and failed tracheal intubation. Br J Anaesth 2008; 100: 116-9. 
3. Theiler LG, Kleine-Brueggeney M, Kaiser D, Urwyler N, Luyet C, Vogt A, et al. Crossover comparison of the laryngeal mask supreme and the i-gel in simulated difficult airway scenario in anesthestized patients. Anesthesiology 2009; 111: 55-62.

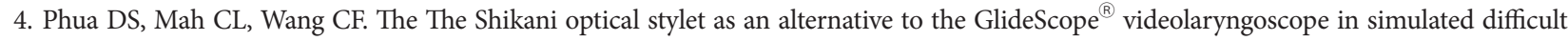
intubations--a randomised controlled trial. Anaesthesia 2012: 67: 402-6.

5. Jeon WJ, Shim JH, Cho SY, Baek SJ. Stylet or forceps-guided tube exchanger to facilitate GlideScope intubation in simulated difficult intubations- a randomized controlled trial. Anaesthesia 2013; 68: 585-90.

6. Kheterpal S, Martin L, Shanks AM, Tremper KK. Prediction and outcomes of impossible mask ventilation: a review of 50,000 anesthestics. Anesthesiology 2009; 110: 891-7.

7. Asai T, Koga K, Vaughan RS. Respiratory compications associated with tracheal intubation and extubation. Br J Anaesth 1998; 80: 767-75.

8. Cattano D, Panicucci E, Paolicchi A, Forfori F, Giunta F, Hagberg C. Risk factors assessment of the difficult airway: an italiansurvey of 1956 patients. Anesth Analg 2004; 99: 1774-9.

9. Combes X, Le Roux B, Suen P, Dumerat M, Motamed C, Sauvat S, et al. Unanticipated airway in anesthetized patients: prospective validation of a management algorithm. Anesthesiology 2004; 100: 1146-50.

10. Savoldelli GL, Schiffer E, Abegg C, Baeriswyl V, Clergue F, Waeber JL. Comparison of the Glidescope, the McGrath, the Airtrap and the Macintosh laryngoscopes in simulated difficult airways. Anaesthesia 2008; 63: 1358-64.

11. Maassen R, Lee R, Hermans B, Marcus M, van Zundert A. A comparison of three videolaryngoscopes: the Macintosh laryngoscope blade reduces, but does not replace routine stylet use for intubation in morbidly obese patients. Anesth Analg 2009; 109: 1560-5.

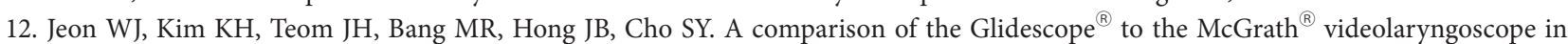
patients. Korean J Anesthesiol 2011; 61: 19-23. 